\title{
Estudo exploratório e confirmatório à estrutura factorial da versão portuguesa do Perception of Success Questionnaire (POSQ).
}

\author{
António Manuel Fonseca \\ Faculdade de Ciências do Desporto e de Educação Física \\ Universidade do Porto \\ Portugal
}

https://doi.org/10.5628/rpcd.01.03.61

\section{RESUMO}

Com esta investigação procurou-se examinar a estrutura factorial da versão portuguesa do Perception of Success Questionnaire (POSQ), designada de Questionário sobre a Percepção de Sucesso no Desporto (POSQp). No primeiro estudo participaram 691 praticantes de diversas modalidades desportivas, com idades compreendidas entre os 13 e os 19 anos de idade. A Análise Factorial Exploratória às suas respostas identificou a existência de dois factores semelhantes não só aos factores propostos para a versão original do instrumento, mas também aos identificados num estudo anterior com a versão portuguesa, designados de Tarefa e Ego. No segundo estudo, participaram 1780 praticantes de diversas modalidade desportivas, com idades compreendidas entre os 13 e os 24 anos de idade.

A Análise Factorial Confirmatória revelou um bom ajustamento do modelo de medida constituído pelos dois factores Tarefa e Ego. Logo, os resultados dos dois estudos realizados providenciaram evidência clara no sentido da validade e fiabilidade do POSQp, razão pela qual se recomenda a sua utilização por parte dos investigadores interessados neste domínio do conhecimento.

Palavras-chave: Motivação, objectivos de realização, avaliação, instrumento, propriedades psicométricas.

\author{
António de Paula Brito \\ Faculdade de Motricidade Humana \\ Universidade Técnica de Lisboa \\ Portugal
}

\begin{abstract}
Exploratory and Confirmatory Study to the Factorial Structure of the Portuguese Version of Perception of Success Questionnaire (Posq).

The purpose of this study was to examine the factorial structure of the Portuguese version of the Perception of Success Questionnaire (POSQ), named "Questionário sobre a Percepção do Sucesso no Desporto (POSQp)". In the first study participated 691 athletes of several sports, aged 13 to 19 years old. Exploratory Factorial Analysis identified two factors similar to the factors proposed for the original version of the instrument as well to the factors identified in a previous study with the Portuguese version, designated Task and Ego. In the second study, participated 1780 athletes of several sports, aged 13 to 24 years old. Confirmatory Factorial Analysis revealed a good fit of the measurement model constituted by Task and Ego factors. Therefore, results of both studies provided clear evidence of validity and reliability of POSQp, reason for that its use is recommended.
\end{abstract}

Keywords: Motivation, achievement goal orientations, assessment, instrument, psychometric properties. 


\section{INTRODUÇÃO}

Ao consultarmos, mesmo que de forma breve, a investigação produzida no domínio da motivação para a prática de actividades de natureza desportiva, não apenas actualmente mas também ao longo dos últimos anos, facilmente constatamos o primado consignado aos estudos decorrentes de uma abordagem conceptual inspirada nas teorias sociocognitivas, particularmente no que se refere ao papel central ocupado pela competência percebida pelos indivíduos para a realização das tarefas com as quais se deparam.

Baseados nos postulados das teorias da motivação para a competência $(19,39)$, os quais destacam que os indivíduos se orientam fundamentalmente para domínios e actividades em relação aos quais se sentem mais competentes, foram publicados nas últimas duas décadas numerosos trabalhos sublinhando a existência de mais do que um critério de sucesso a partir do qual são construídas as percepções dos indivíduos acerca da sua competência, de entre os quais podemos destacar os protagonizados por Nicholls $(23,24,25,26)$, Ames $(1,2)$, Dweck $(7,8)$ ou Maher e Braskamp (21).

De acordo com a perspectiva subscrita por estes autores, os indivíduos são assumidos como organismos intencionais e orientados por objectivos, designados por objectivos de realização, porquanto se constituem como os objectivos que os indivíduos procuram realizar e para os quais orientam os seus comportamentos. Estes objectivos operam de forma racional, influenciando e sendo influenciados pelas suas crenças de realização, e orientam as tomadas de decisão e comportamentos que ocorrem em contextos de realização.

Ainda que subsista alguma divergência quanto ao número exacto de critérios de sucesso, ou objectivos de realização, normalmente adoptados pelos indivíduos em contextos desportivos, os quais podem inclusivamente variar com a sua idade, de uma forma geral considera-se a existência de dois principais: um centrado na Tarefa e outro no Ego.

De sublinhar contudo, a este propósito, que também em relação à designação dos critérios de sucesso não existe completo consenso. Ou seja, apesar das designações Tarefa e Ego, propostas por Nicholls (23, 24,
$25,26)$, serem provavelmente as adoptadas mais frequentemente no domínio da investigação sobre os objectivos de realização no desporto - razão pela qual decidimos adoptá-las ao longo desta nossa pesquisa - há quem prefira adoptar outras designações (para mais detalhes, ver 11).

Independentemente da adopção de uma outra designação para aqueles critérios, permanece todavia o consenso de que em associação ao primeiro deles se encontra uma análise autoreferenciada do rendimento e a procura da demonstração de mestria na realização de uma determinada tarefa. Do mesmo modo, relativamente ao segundo, persiste a unanimidade de que associada a ele emerge uma comparação normativa do rendimento por parte do próprio indivíduo e a procura de demonstração de habilidade quando em comparação com outros indivíduos.

Para a avaliação dos objectivos de realização dos indivíduos em contextos desportivos têm sido desenvolvidos nos últimos anos diversos instrumentos (para mais detalhes, ver 29,30), de entre os quais o mais utilizado é indiscutivelmente o Task and Ego Orientation in Sport Questionnaire (TEOSQ), desenvolvido em 1989 por Joan Duda (actualmente uma das principais referências neste domínio do conhecimento) e o seu orientador John Nicholls (5). Porém, considerando que o TEOSQ, não obstante a demonstração de aceitáveis índices de validade e fiabilidade, resulta da adaptação de um instrumento desenvolvido em contextos de natureza educativa para contextos de natureza desportiva, Glyn Roberts e Glória Balagué (também eles duas das referências mais reconhecidas nesta área), igualmente por volta dos finais dos anos 80 , decidiram desenvolver um instrumento de avaliação dos objectivos de realização específico para situações desportivas, que designaram de Perception of Success Questionnaire (POSQ; 32, 33, 34, 35).

O POSQp é constituído pela descrição de 12 situações passíveis de ocorrer em contextos desportivos, relativamente a cada uma das quais o inquirido indica o modo como considera que se sentiria bem sucedido se ela ocorresse efectivamente com ele, através do recurso a uma escala de Likert de 5 pontos (de $1=$ discordo totalmente a $5=$ concordo totalmente). Enquanto seis das situações se relacio- 
nam com a Tarefa (e.g., 'sinto-me com mais sucesso quando demonstro clara melhoria no meu rendimento') as outras seis referem-se ao Ego (e.g., 'sinto-me com mais sucesso quando derroto os meus adversários').

Após a sua publicação, o POSQ tem vindo a ser referido por diversos investigadores como possuidor de boas qualidades psicométricas, seja no que se refere à versão original $(32,33,34,35)$, seja relativamente a versões traduzidas noutras línguas (por exemplo, $4,9,36)$.

Daí que, em 1995 (12), tenha sido decidido iniciar o processo da sua adaptação e validação transcultural para a língua e cultura portuguesas, com os resultados dos estudos subsequentes $(13,14,15,16)$ a revelarem-se como promissores, porquanto permitiram verificar que a estrutura factorial identificada através do recurso à Análise Factorial Exploratória (AFE) foi similar à proposta para a versão original. Adicionalmente, e correspondendo à sugestão da necessidade de aprofundamento do estudo das propriedades psicométricas do POSQp, designadamente no que concerne à utilização da Análise Factorial Confirmatória (AFC) $(14,16)$, Fonseca, Maia, Garganta e Biddle $(17,18)$ recorreram àquela técnica estatística para analisar a estrutura factorial anteriormente identificada e a sua invariância estrutural ao longo da idade de um grupo de praticantes de diversas modalidade desportivas do sexo feminino.

Em termos gerais, os resultados encontrados providenciaram suporte empírico no sentido da sugestão da validade e fiabilidade do POSQp. Ainda assim, atendendo a que foram detectados alguns ligeiros problemas no ajuste global do modelo de medida examinado, foi recomendada a realização de trabalho adicional, razão pela qual foi desenvolvida a presente investigação.

\section{MATERIAL E MÉTODOS}

\section{Amostra}

Estudo 1

Participaram neste estudo 691 praticantes de diversas actividades físicas ou desportivas (andebol, atletismo, basquetebol, futebol e actividades de academia), de ambos os sexos (164 do sexo feminino e 527 do sexo masculino), com idades compreendidas entre os 13 e os 19 anos (16.35 \pm 3.57 anos).

\section{Estudo 2}

Participaram neste estudo 1780 praticantes de diversas actividades físicas ou desportivas (actividades de academia, andebol, atletismo, basquetebol, futebol, hóquei em patins, natação, polo aquático, rugby e voleibol), de ambos os sexos (692 do sexo feminino e 1088 do sexo masculino), com idades compreendidas entre os 13 e os 24 anos (16.34 \pm 2.86 anos).

\section{Procedimentos de aplicação}

Todos os participantes preencheram o POSQp, no âmbito de um estudo mais abrangente sobre as determinantes motivacionais da sua actividade física ou desportiva, antes ou depois de um dos seus treinos ou aulas. Não foram detectadas quaisquer dificuldades de compreensão relativamente ao modo de preenchimento ou ao significado dos itens constituintes do instrumento.

\section{Procedimentos estatísticos}

Os procedimentos estatísticos utilizados ao longo dos dois estudos apresentados em seguida foram: média, desvio-padrão, assimetria, achatamento, análise factorial exploratória, coeficiente alfa de Cronbach e análise factorial confirmatória. Os programas estatísticos utilizados foram o SPSS (versão 10.0) e o LISREL (versão 8.30).

\section{RESULTADOS}

\section{Estudo 1}

A análise descritiva dos resultados (ver Quadro 1) revelou que os inquiridos utilizaram, para cada um dos 12 itens, as cinco possibilidades de resposta existentes, com os valores médios a variarem entre 3.00 e 4.50; foi ainda evidente que os valores atribuídos aos diferentes itens obedeceram a uma distribuição normal univariada, porquanto não foram detectados quaisquer fenómenos de assimetria ou achatamento. 
Quadro 1. Estatística descritiva das respostas ao POSQp.

\begin{tabular}{lcccc} 
item & Mín.-Máx. & M d.p. & Assimetria & Achatamento \\
\hline 01 ...derroto outras pessoas & $1-5$ & $3.13 \pm 1.35$ & 0.05 & -1.10 \\
02 ...sou claramente superior & $1-5$ & $3.12 \pm 1.33$ & 0.07 & -1.18 \\
03 ...sou o melhor & $1-5$ & $3.00 \pm 1.38$ & 0.11 & -1.24 \\
04 ...trabalho no duro & $1-5$ & $4.28 \pm 0.98$ & -1.12 & 1.11 \\
05 ...demonstro clara melhoria no meu rendimento & $1-5$ & $4.34 \pm 0.81$ & -1.09 & 0.92 \\
06 ...rendo mais do que os meus adversários & $1-5$ & $3.76 \pm 1.12$ & -0.51 & -0.55 \\
07 ...atinjo um objectivo & $1-5$ & $4.49 \pm 0.75$ & -1.63 & 1.95 \\
08 ...ultrapasso dificuldades & $1-5$ & $4.50 \pm 0.72$ & -1.39 & 1.89 \\
$09 . .$. atinjo objectivos pessoais & $1-5$ & $4.20 \pm 0.93$ & -1.06 & 0.78 \\
$10 . .$. ganho & $1-5$ & $3.93 \pm 1.06$ & -0.73 & -0.03 \\
$11 \ldots$ mostro às outras pessoas que sou o melhor & $1-5$ & $3.02 \pm 1.25$ & 0.04 & -0.91 \\
12 ...rendo ao nível máximo das minhas capacidades & $1-5$ & $4.48 \pm 0.79$ & -1.50 & 1.84
\end{tabular}

As rotações varimax e oblimin da AFE produziram resultados bastante similares, razão pela qual se apresentam apenas os referentes à rotação varimax (ver quadro 2) a qual, de um modo geral, permite a extracção de uma solução factorial mais clara (30).

Quadro 2. Resultados da Análise Factorial Exploratória [rotação varimax) ao POSQp.

\begin{tabular}{|c|c|c|}
\hline Item & Ego & Tarefa \\
\hline 01 ...derroto outras pessoas & .69 & \\
\hline 02 ...sou claramente superior & .84 & \\
\hline 03 ...sou o melhor & .81 & \\
\hline 06 ...rendo mais do que os meus adversários & .59 & \\
\hline 10 ...ganho & .59 & \\
\hline 11 ...mostro às outras pessoas que sou o melhor & .79 & \\
\hline 04 ...trabalho no duro & & .52 \\
\hline 05 ...demonstro clara melhoria no meu rendimento & & .75 \\
\hline 07 ...atinjo um objectivo & & .78 \\
\hline 08 ...ultrapasso dificuldades & & 77 \\
\hline 09 ...atinjo objectivos pessoais & & .61 \\
\hline 12 ...rendo ao nível máximo das minhas capacidades & & .64 \\
\hline Valor próprio & 4.32 & 2.06 \\
\hline \% variância & 36.0 & 17.2 \\
\hline
\end{tabular}

Foram identificados dois factores, não correlacionados entre si $(\mathrm{r}=0.09 ; \mathrm{p}>.05)$, com valores próprios superiores a 1.0, responsáveis, no seu conjunto, por $53 \%$ da variância total das respostas dos indivíduos, e o valor mínimo de saturação dos itens no respectivo factor foi de 0.52 .
No que concerne à consistência interna dos itens do POSQp (ver Quadro 3), verificámos que os elevados valores do alfa de Cronbach para cada um dos factores (0.84 e 0.78 , respectivamente para Ego e Tarefa) não poderiam ser aumentados a partir da eliminação de qualquer dos seus itens. Em relação às corre- 
lações item-factor, a análise dos resultados revelou que quase todas elas foram superiores a 0.45 ; a única excepção foi constituída pelo item 4 , que ainda assim apresentou uma correlação de 0.39 com o factor respectivo.

Quadro 3. Consistência interna dos itens das dimensões do POSQp.

$\begin{array}{ll}.52 & .84 \\ .74 & .78 \\ .70 & .79 \\ .56 & .82 \\ .53 & .83 \\ .65 & .80\end{array}$

02 ...sou claramente superior

03 ...sou o melhor

06 ...rendo mais do que os meus adversários

10 ...ganho

11 ...mostro às outras pessoas que sou o melhor

04 ...trabalho no duro

05 ...demonstro clara melhoria no meu rendimento

07 ...atinjo um objectivo

08 ...ultrapasso dificuldades

09 ...atinjo objectivos pessoais

\section{Estudo 2}

O modelo de medida do POSQp submetido à AFC (ver Figura 1) foi rigorosamente idêntico ao modelo de medida proposto pelos autores da versão original do instrumento. Ou seja, postulámos a existência de dois factores (Ego e Tarefa), constituídos cada um por seis itens (Ego: itens 1, 2, 3, 6, 10 e 11; Tarefa: itens 4, 5, 7, 8, 9 e 12).

A análise dos resultados permitiu verificar que, não obstante o valor elevado e estatisticamente significativo do $\chi^{2}\left(\chi_{53}^{2}=903.82, \mathrm{p}<.001\right)$, o valor dos índices considerados para determinar a bondade do ajustamento global do modelo de medida do POSQp inspeccionado foi igual (AGFI $=0.90$ e $\mathrm{CFI}=0.90$ ) ou superior $(\mathrm{GFI}=0.93)$ ao valor critério de 0.90 ; relativamente à RMSR o valor foi de 0.06 .
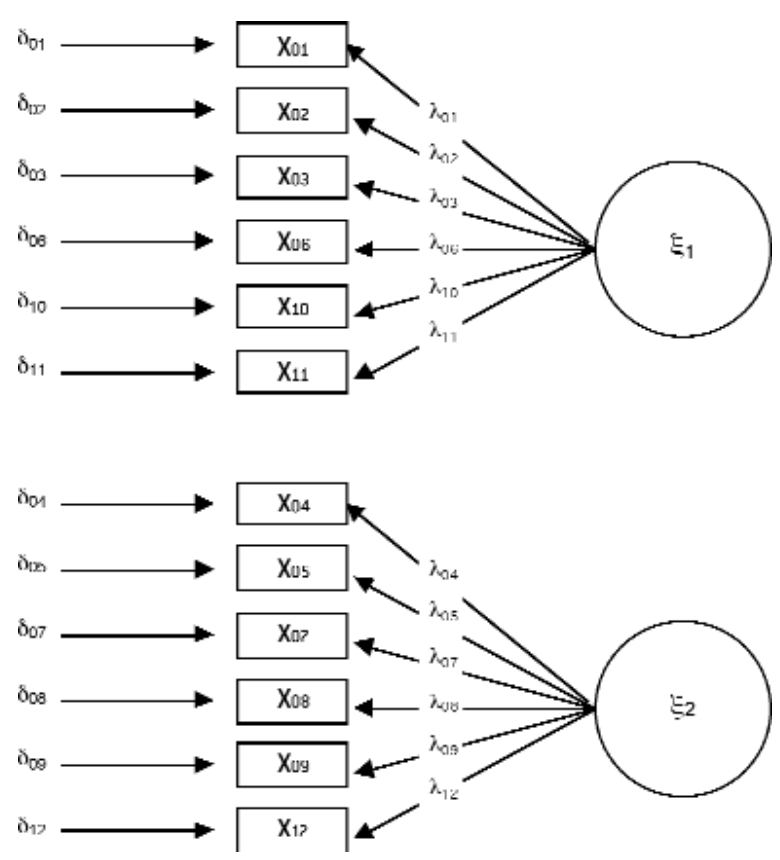
Quanto aos parâmetros individuais (ver Quadro 4), todos os itens saturaram nos dois factores no sentido postulado, com uma magnitude superior a 0.50 ; os valores $t$ foram todos elevados e estatisticamente significativos.

Quadro 4. Estimativas dos parâmetros estandardizados do POSQp, valores te $p$.

\begin{tabular}{|c|c|c|c|c|}
\hline Item & Ego & Tarefa & $t$ & $\mathrm{p}$ \\
\hline 01 ...derroto outras pessoas & .62 & & 29.20 & $<.001$ \\
\hline 02 ...sou claramente superior & .85 & & 44.82 & $<.001$ \\
\hline 03 ...sou o melhor & .83 & & 43.06 & $<.001$ \\
\hline 04 ...trabalho no duro & & .51 & 21.52 & $<.001$ \\
\hline 05 ...demonstro clara melhoria no meu rendimento & & .68 & 31.22 & $<.001$ \\
\hline 06 ...rendo mais do que os meus adversários & .61 & & 28.24 & $<.001$ \\
\hline 07 ....atinjo um objectivo & & .70 & 32.14 & $<.001$ \\
\hline 08 ...ultrapasso dificuldades & & .69 & 31.75 & $<.001$ \\
\hline 09 ...atinjo objectivos pessoais & & .51 & 21.97 & $<.001$ \\
\hline 10 ...ganho & .51 & & 23.14 & $<.001$ \\
\hline 11 ...mostro às outras pessoas que sou o melhor & 70 & . & 34.25 & $<.001$ \\
\hline 12 ...rendo ao nível máximo das minhas capacidades & & .63 & 27.90 & $<.001$ \\
\hline
\end{tabular}

\section{DISCUSSÃO E CONCLUSÕES}

Os resultados da AFE, para além de terem sido, na sua globalidade, bastante semelhantes aos referidos em estudos similares, realizados quer com a versão original do POSQ $(22,32,33,34,35,37,38)$ quer com versões traduzidas noutras línguas $(6,10)$, sugeriram a superioridade da versão do POSQp agora testada relativamente a uma versão inicial testada anteriormente $(13,14,15,16)$. De facto, no presente estudo, não só os dois factores identificados foram perfeitamente idênticos aos factores propostos pelos autores da versão original, como o valor da percentagem de variância explicada foi extremamente semelhante ao referido por Roberts e colaboradores $(32,33,34,35,37,38)$ e claramente superior ao que havíamos encontrado anteriormente (13, $14,15,16)$.

De sublinhar igualmente que, no presente estudo, ao contrário do verificado com a versão preliminar do POSQp $(13,14,15,16)$, não foram detectados quaisquer indícios da existência de valores de saturação cruzada dos itens; ou seja, todos os itens saturaram de forma elevada no factor em que era suposto saturarem e de forma bastante reduzida no outro factor. Para além disso, os valores de saturação dos itens nos respectivos factores foram, a exemplo do registado por outros investigadores $(6,32,33,34$, $35)$, bastante superiores aos valores sugeridos na literatura $(3,31)$ como aceitáveis para se proceder à sua retenção.

Também ao analisarmos os valores relativos à consistência interna dos itens dos dois factores do POSQp verificámos que ambos foram substancialmente superiores ao valor mínimo de 0.70 proposto por Nunnally (27), aproximando-se dos valores apresentados nos estudos de Roberts e colaboradores $(32,33,34,35,37,38)$.

Finalmente, em consonância não só com o sugerido por Nicholls $(23,24,25,26)$, e posteriormente constatado por Roberts e colaboradores $(32,33,34,35$, $37,38)$, mas também com os resultados de estudos anteriormente realizados entre nós $(13,14,15,16)$, foi evidente a inexistência de uma correlação significativa entre os valores relativos aos dois factores, reflectindo, por isso mesmo, a ortogonalidade existente entre os dois critérios de sucesso examinados. Os resultados da AFC ao modelo de medida do POSQp permitiram aquilatar da inequívoca qualidade do seu ajustamento global e individual aos dados empíricos analisados. Se, por exemplo, compararmos 
os resultados deste estudo com os encontrados por Durand e colaboradores (6) para a versão francesa do POSQ detectamos a existência de uma certa semelhança: estes investigadores, após terem procedido à reespecificação do modelo de medida inspeccionado (considerando a existência de erros de medida correlacionados entre alguns dos itens) encontraram, respectivamente para uma amostra de indivíduos adultos e outra de jovens, os valores de 0.95 e 0.94 para o GFI, 0.92 e 0.91 para o AGFI e de 0.06 e 0.06 para a RMSR; a estatística do $\chi^{2}$, tanto num caso como noutro, produziu valores estatisticamente significativos. De recordar que, no presente estudo, mesmo sem havermos procedido a qualquer reespecificação do modelo de medida, encontrámos valores de 0.93 para o GFI, de 0.90 para o AGFI e de 0.06 para a RMSR, os quais foram igualmente superiores aos reportados num estudo anteriormente realizado com o POSQp $(17,18)$.

Também relativamente à versão original foi, por exemplo, possível constatar que o valor da RMSR do modelo de medida do POSQp foi ligeiramente mais baixo do que os encontrados em dois estudos recentemente realizados nos Estados Unidos da América. Mais especificamente, enquanto Kavussanu (20) referiu no seu estudo ter encontrado um valor de 0.09 para aquele indicador, Roberts e colaboradores (35) reportaram 0.07.

No que concerne aos índices de bondade do ajustamento do modelo, não é possível proceder a uma comparação da mesma natureza, porquanto estes investigadores não optaram pelos índices CFI, GFI ou AGFI, mas sim pelo índice Tucker-Lewis. Ainda assim, há que referir que o valor de 0.90 do índice Tucker-Lewis encontrado naqueles dois estudos se pode considerar, em certa medida, semelhante aos valores dos indicadores de ajustamento global seleccionados para o presente estudo.

Em suma, os resultados dos estudos exploratório e confirmatório que realizámos pareceram revelar que as propriedades psicométricas do POSQp podem ser já consideradas como claramente satisfatórias, razão pela qual se recomenda a sua utilização para a avaliação dos objectivos de realização perseguidos pelos jovens portugueses no âmbito da sua prática de uma actividade física ou desportiva.

\section{CORRESPONDÊNCIA}

António Manuel Fonseca

Gabinete de Psicologia do Desporto

Faculdade de Ciências do Desporto

e de Educação Física

Rua Dr. Plácido Costa, 91

4200 Porto

afonseca@fcdef.up.pt 


\section{REFERÊNCIAS BIBLIOGRÁFICAS}

1. Ames, C. (1992). Achievement goals, motivation climate and motivational processes. In G.C. Roberts (Ed.), Motivation in sport and exercise. Champaign, IL: Human Kinetics, 161-176. 2. Ames, C. \& Archer, J. (1987). Mothers' beliefs about the role of ability and effort in school learning. Journal of Educational Psychology, 19:409-414.

3. Camacho, J. (1995). Análisis multivariado com SPSS/PC+. Barcelona: EUB.

4. Cury, F., Sarrazin, P. \& Famose, J.P. (1994). Mesurer différents aspects de la motivation d'accomplissement en sport: Propriétés psychométriques du SAGQ de l'EPCM et de l'IMI. In M.Audiffren \& G.Minvielle (Eds), Psychologie des pratiques physiques et sportives. Université de Poitiers: Presses de l'UFR APS, 299-300.

5. Duda, J.L. \& Nicholls, J.G. (1989). The Task and Ego Orientation in Sport Questionnaire. Trabalho não publicado. Universidade de Purdue.

6. Durand, M., Cury, F., Sarrazin, P. \& Famose, J.P. (1996). Le Questionnaire de Perception du Succès en Sport: Validation française du "Perception of Success Questionnaire". International Journal of Sport Psychology, 27:251-268.

7. Dweck, C.S. (1986). Motivational processes affecting learning. American Psychologist, 41:1040-1048.

8. Dweck, C.S. \& Elliott, E. (1983). Achievement motivation. In H. Hetherington (Ed.), Handbook of child psychology: Socialization, personality and social development. New York: John Wiley \& Sons, 4th ed., vol. 4, 643-691.

9. Escarti, A. \& Cervelló, E. (1994, Julho). Relationship between the subjective perception of the achievement goals orientation of the 'other significatives' and the own orientation: A spanish perspective. Trabalho apresentado no 23rd International Congress of Applied Psychology. Madrid, Espanha.

10. Escarti, A., Cervelló, E. \& Guzman, J.F. (1996). La orientación de metas de adolescentes deportistas de competición y la percepción de los critérios de exito deportivo de los otros significativos. Revista de Psicologia Social Aplicada, 6:27-42.

11. Fonseca, A.M. (1999). Atribuiçôes em contextos de actividade física ou desportiva: Perspectivas, relaçóes e implicaçóes. Dissertação de doutoramento não publicada. Porto: FCDEF, Universidade do Porto.

12. Fonseca, A.M. \& Balagué, G. (1995). Versão portuguesa do Perception of Success Questionnaire (POSQ): Questionário sobre a Percepção de Sucesso no Desporto (POSQp). Trabalho não publicado. FCDEF, Universidade do Porto.

13. Fonseca, A.M. \& Balagué, G. (1996a). Measuring goal orientations in youth competitive soccer: A comparison of TEOSQ and POSQ measures. Journal of Applied Sport Psychology, 8 (supplement): S143.

14. Fonseca, A.M. \& Balagué, G. (1996b). Perception of Success Questionnaire (POSQ): Estudo preliminar sobre a validade da sua versão portuguesa. Trabalho apresentado na IV Conferência Internacional "Avaliação Psicológica: Formas e contextos" \& II Mostra de "Provas Psicológicas em Portugal". Universidade do Minho, Braga, Portugal.

15. Fonseca, A.M. \& Balagué, G. (2001a). Avaliação dos objectivos de realização no futebol juvenil de competição:

Comparação entre o TEOSQp e o POSQp. In António Fonseca (Ed.), A FCDEF-UP e a Psicologia do Desporto: Estudos sobre motivação. Porto: FCDEF, Universidade do Porto, 59-61.

16. Fonseca, A.M. \& Balagué, G. (2001b). Perception of Success Questionnaire (POSQ): Estudo preliminar sobre a vali- dade da sua versão portuguesa. In António Fonseca (Ed.), A FCDEF-UP e a Psicologia do Desporto: Estudos sobre motivação. Porto: FCDEF, Universidade do Porto, 68-70.

17. Fonseca, A.M., Maia, J.A., Garganta, R. \& Biddle, S.J.H. (1998). Análise à invariância estrutural da versão portuguesa do Perception of Success Questionnaire: Um estudo com atletas do sexo feminino de diferentes idades. Trabalho apresentado na VI Conferencia Internacional de Evaluación Psicológica.

Universidad de Salamanca, Espanha.

18. Fonseca, A.M., Maia, J.A., Garganta, R. \& Biddle, S.J.H. (2001). Análise à invariância estrutural da versão portuguesa do Perception of Success Questionnaire: Um estudo com atletas do sexo feminino de diferentes idades. In António Fonseca (Ed.), A FCDEF-UP e a Psicologia do Desporto: Estudos sobre motivação. Porto: FCDEF, Universidade do Porto, 95.

19. Harter, S. (1978). Effectance motivation reconsidered: Toward a developmental model. Human Development, 21: 34-64. 20. Kavussanu, M. (1996). Moral development and behaviour in sport: An achievement goal perspective. Dissertação de doutoramento não publicada. University of Illinois, Estados Unidos da América.

21. Maher, M. \& Braskamp, L. (1986). The motivation factor: $A$ theory of personal investment. Lexington MA: Lexington Books. 22. Marsh, H.W. (1994). Sport motivation orientations: Beware of jingle-jangle fallacies. Journal of Sport \& Exercise Psychology, $16: 365-380$

23. Nicholls, J. (1984a). Achievement motivation: Conceptions of ability, subjective experience, task choice, and performance. Psychological Review, 91:328-346.

24. Nicholls, J. (1984b). Conceptions of ability and achievement motivation. In R.Ames \& C.Ames (Eds), Research on motivation in education (vol. 1). New York: Academic Press.

25. Nicholls, J. (1989). The competitive ethos and democratic education. Cambridge, MA: Harvard University Press.

26. Nicholls, J. (1992). The general and the specific in the development and expression of achievement motivation. In G.C. Roberts (Ed.), Motivation in sport and exercise. Champaign, IL: Human Kinetics, 31-56.

27. Nunnally, J.C. (1978). Psychometric theory (2nd edition). St.Louis: McGraw-Hill.

28. Ostrow, A.C. (1990). Directory of psychological tests in the sport and exercise sciences. Morgantown, WV: Fitness Information Technology Inc.

29. Ostrow, A.C. (1996). Directory of psychological tests in the sport and exercise sciences (2nd edition). Morgantown, WV: Fitness Information Technology Inc.

30. Pasquali, L (1994). Curso de Análise Factorial e Análise da Variância. Colégio Universitário de Altos Estudos, Universidade do Porto.

31. Pedhazur, E.L. (1982). Multiple regression in behavioral research - Explanation and prediction. Fort Worth: Holt, Rinehart \& Winston.

32. Roberts, G.C. \& Balague, G. (1989). The development of a social cognitive scale of motivation. Trabalho apresentado no VII World Congress of Sport Psychology. Singapura.

33. Roberts, G.C. \& Balague, G. (1991). The development and validation of the Perception of Success Questionnaire. Trabalho apresentado no VIII European Congress of the FEPSAC. Colónia, Alemanha.

34. Roberts, G.C., Treasure, D., \& Balague, G. (1995). The development and validation of the Perception of Success Questionnaire. Proceedings of the IXth European Congress on Sport Psychology. Brussels: FEPSAC, 75-81. 
35. Roberts, G.C., Treasure, D.C., \& Balague, G. (1998).

Achievement goals in sport: The development and validation of the Perception of Success Questionnaire. Journal of Sport Sciences, 16:337-347.

36. Sarrazin, P. Famose, J.P., Biddle, S.J.H., Fox, K., Durand, M. \& Cury, F. (1995). Buts d'acomplissement et croyances relatives à la nature de l'habilité motrice. Science \& Motricité, 26:2131.

37. Treasure, D. \& Roberts, G. (1994a). Cognitive and affective concomitants of task and ego goal orientations during the middle-school years. Journal of Sport \& Exercise Psychology, 16 (1):15-28.

38. Treasure, D. \& Roberts, G. (1994b). Perception of Success Questionnaire: Preliminary validation in an adolescent population. Perceptual and Motor Skills, 79:607-610.

39. White, R.W. (1959). Motivation reconsidered: The concept of competence. Psychological Review, 66 (5):297-333. 